\title{
Nadofaragene Firadenovec/Syn3
}

National Cancer Institute

\section{Source}

National Cancer Institute. Nadofaragene Firadenovec/Syn3. NCI Thesaurus. Code C104743.

A non-replicating recombinant adenovirus type 5 (Ad5)-vector encoding the gene for interferon alpha-2b (IFN alpha-2b) and the gene transfer enhancement agent Syn 3, with potential antineoplastic activity. Upon intravesical administration, nadofaragene firadenovec/Syn3 transfects both cancerous and normal bladder cells, and the adenovirus secretes interferon (IFN alpha-2b) into the bladder. IFN exerts a direct antitumor killing effect and a bystander effect, thereby killing adjacent, non-transfected cancerous bladder cells. Syn 3 enhances the ability of the adenoviral vector to transfect cells. 